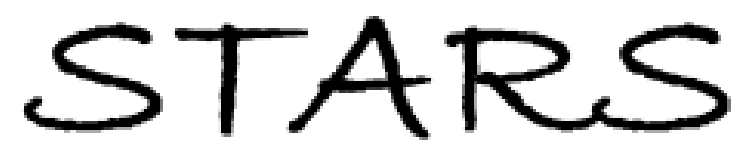

University of Central Florida

STARS

Faculty Bibliography 2000s

Faculty Bibliography

$1-1-2005$

\title{
Tera-sample per second real-time waveform digitizer
}

Yan Han

Ozdal Boyraz

Bahram Jalali

Find similar works at: https://stars.library.ucf.edu/facultybib2000

University of Central Florida Libraries http://library.ucf.edu

This Article is brought to you for free and open access by the Faculty Bibliography at STARS. It has been accepted for inclusion in Faculty Bibliography 2000s by an authorized administrator of STARS. For more information, please contactSTARS@ucf.edu.

\section{Recommended Citation}

Han, Yan; Boyraz, Ozdal; and Jalali, Bahram, "Tera-sample per second real-time waveform digitizer" (2005). Faculty Bibliography 2000s. 5251.

https://stars.library.ucf.edu/facultybib2000/5251

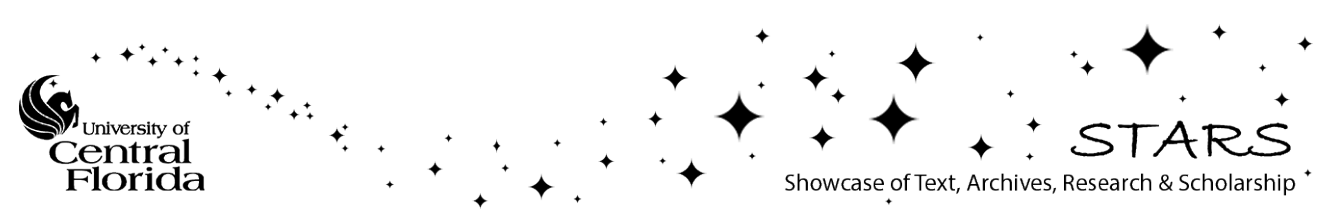




\title{
Tera-sample per second real-time waveform digitizer
}

\author{
Yan Han, ${ }^{\text {a) }}$ Ozdal Boyraz, and Bahram Jalali \\ Department of Electrical Engineering, University of California, Los Angeles, California 90095
}

(Received 7 April 2005; accepted 17 October 2005; published online 8 December 2005)

\begin{abstract}
We demonstrate a real-time transient waveform digitizer with a record 1 tera-sample per second sampling rate. This is accomplished by using a photonic time stretch preprocessor which slows down the electrical waveform before it is captured by an electronic digitizer. (C) 2005 American Institute of Physics. [DOI: 10.1063/1.2142087]
\end{abstract}

Digital processing and storage of information is a ubiquitous function encountered in virtually every science and engineering discipline. Since signals originating in the physical world are analog, analog-to-digital converters (ADC), otherwise known as digitizers, play a central role. The most challenging signals to digitize are ultrafast transients that are nonrepetitive. These waveforms arise in a number of applications including (i) diagnostic tool in particle accelerators that probe fundamental building blocks of nature; (ii) x-ray free electron lasers; ${ }^{1}$ (iii) study of electromagnetic pulse weapons. ${ }^{2}$ Digitizers with real-time capability are required because the nonrepetitive nature of these signals renders traditional sampling oscilloscopes useless.

The sampling rate of conventional electronic ADCs, such as ones used in high speed instruments, is limited by the speed of the electronic circuitry, and in the case where the interleaving architecture is used, by the mismatches in the multichannel ADC array. ${ }^{3,4}$ While the performance of electronic ADCs continues to improve, the sampling rate of a state-of-the-art system is currently about $20 \mathrm{GSa} / \mathrm{s}$ with $\sim 5$ effective number of bits (ENOB). Achieving tera-sample per second $(\mathrm{TSa} / \mathrm{s})$ performance is clearly beyond the reach of conventional approaches. One potential solution to overcome the electronic bottleneck is to use photonic preprocessing. In particular, the photonic time stretch approach has proven to be an effective way to extend the sampling rate and the bandwidth. Here, the high speed transient waveform is first slowed down and then captured by a conventional electronic digitizer. ${ }^{5,6}$ In this letter, we demonstrate such a system that achieves a sampling rate of $1 \mathrm{TSa} / \mathrm{s}$. This system consists of a $50 \times$ time stretch preprocessor and a $20 \mathrm{GSa} / \mathrm{s}$ electronic digitizer. To the best of our knowledge, this is the first time that the real-time digitization at $1 \mathrm{TSa} / \mathrm{s}$ has been achieved.

The time stretch preprocessing, shown in Fig. 1, consists of three steps: time-to-wavelength transformation, wavelength domain processing, and wavelength-to-time transformation. Time-to-wavelength transformation occurs when the electrical signal modulates the intensity of a linearly chirped optical pulse. At the output of modulator, the input signal's time scale is linearly mapped onto the optical wavelength. The second and third steps occur simultaneously when the waveform is broadened as it travels through the second dispersive optical medium and is subsequently photodetected.

The major obstacle to achieving a stretch factor of 50 $X$ is to overcome the frequency fading that is associated with

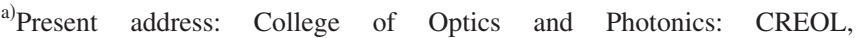
University of Central Florida, Orlando, FL 32816; electronic mail: yanhan@creol.ucf.edu
}

dispersive propagation. This phenomenon has been described in details elsewhere. ${ }^{5,6}$ Briefly, it occurs due to the dispersion-induced interference between the two modulation sidebands. We overcome this problem using the recently proposed phase diversity technique, where two stretched waveforms with complementary fading characteristics are realized and combined to eliminate the bandwidth limitation. ${ }^{7}$ Another practical concern is the large loss of dispersive fiber required to achieve such a large stretch factor. This problem is mitigated by the judicious use of optical amplification in such a manner as to optimize the overall signal to noise ratio while avoiding degradation from optical nonlinearity.

The block diagram of experimental setup for a $50 \times$ photonic time stretch preprocessor is shown in Fig. 2. Ultrashort and broadband optical pulses are produced, with repetition period $T=200 \mathrm{~ns}$, through the continuum generation process. Around $15 \mathrm{~nm}$ of the optical spectrum (centered at $1590 \mathrm{~nm}$ ) is sliced using an optical bandpass filter and is used in the experiment. After being linearly chirped by propagation in a spool of dispersive fiber, a given pulse enters the electrooptic modulator where it captures the electrical transient. The chirped optical pulse has finite amplitude variations resulting in distortion of the captured signal. The distortion is removed by using a reference waveform and digital filtering. ${ }^{6}$ The modulator has two outputs with complimentary fading characteristics, and after delaying one by $T / 2$, the pulse trains at two outputs are time-interleaved and stretched together in the second dispersive fiber. The first fiber has a total dispersion of $D_{1}=-101 \mathrm{ps} / \mathrm{nm}$, creating a chirped optical pulse with around 1.5 ns duration. The second fiber has a total dispersion of $D_{2}=-4921 \mathrm{ps} / \mathrm{nm}$. The stretch factor is give by $\left(D_{1}+D_{2}\right) / D_{1}=50 .^{5,6}$ To minimize the noise contributed by the optical amplifiers, the waveform is filtered by an optical bandpass filter before photodetection. The detected waveform is subsequently captured by a Tektronix TDS7404 (4 GHz analog bandwidth, $20 \mathrm{GSa} / \mathrm{s}$ ) real-time digitizer. The effective sampling rate of this system is $20 \mathrm{GSa} / \mathrm{s}$ $\times 50=1 \mathrm{TSa} / \mathrm{s}$ and the intrinsic input analog bandwidth is $4 \mathrm{GHz} \times 50=200 \mathrm{GHz}$, although, in the experiments re-

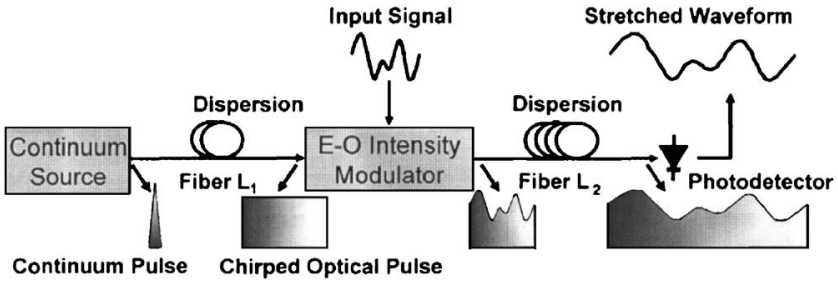

FIG. 1. Block diagram of the photonic time stretch preprocessor. 


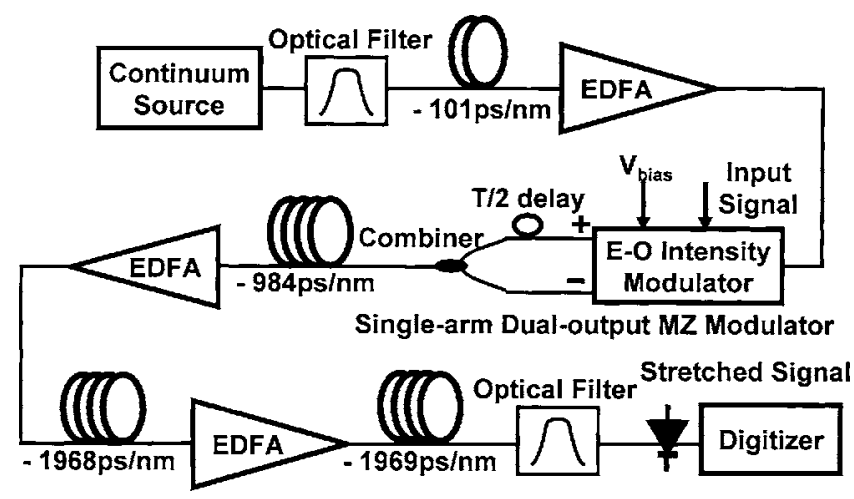

FIG. 2. Experiment setup of $\mathrm{TSa} / \mathrm{s}$ digitizer with a $50 \times$ photonic time stretch preprocessor. EDFA: erbium-doped fiber amplifier.

ported here, the Mach-Zehnder (MZ) electro-optic modulator places a limit of around $50 \mathrm{GHz}$. Figure 3 shows the realtime capture of a $48 \mathrm{GHz}$ tone at $1 \mathrm{TSa} / \mathrm{s}$. The measured voltage full width at half maximum (FWHM) time aperture is $1.1 \mathrm{~ns}$. The time aperture determines the length of transients that can be captured. The measured stretch factor is 50.5. To obtain the average signal-to-noise ratio (SNR), 200 such real-time measurements are preformed. Over a $10 \mathrm{GHz}$ digitally filtered bandwidth centered at $48 \mathrm{GHz}$, the mean and standard deviation of the SNR in each of 200 measurements is $22.7 \pm 1.08 \mathrm{~dB}$ corresponding to $3.5 \pm 0.2$ ENOB. Measurements at other input frequencies with the same bandwidth resulted in up to 4.2 ENOB.

The digitizer performance is also measured in the frequency domain. Figure 4 shows the digital spectrum of a measured $26 \mathrm{GHz}$ signal and a $42 \mathrm{GHz}$ signal, respectively. The Hanning window was used before discrete Fourier transform. We note that no digital filter was used to obtain the spectrum in Fig. 4, hence, the input analog bandwidth of these spectrums is $0-200 \mathrm{GHz}$. The second harmonic distortion tone is observed in Fig. 4. These arise due to nonlinear electro-optic modulation that is affected by memory effects in the fiber, a phenomenon that has been previously predicted by theory. ${ }^{6}$ Postnonlinear compensation performed in the digital domain similar to broadband power amplifier linearization in wireless communications can potentially mitigate this type of distortion. The highest spurious peak appearing at $125 \mathrm{GHz}$ is due to the electronic digitizer and is independent of the time stretch processor.

Although the $\mathrm{TSa} / \mathrm{s}$ digitizer has an intrinsic bandwidth of $200 \mathrm{GHz}$, the experimental input bandwidth is limited by

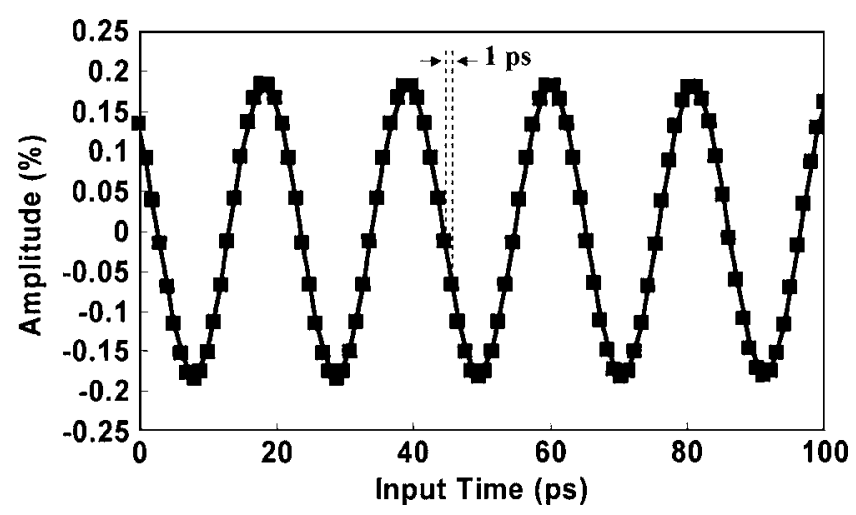

FIG. 3. Digitization of a $48 \mathrm{GHz}$ sinusoid at $1 \mathrm{TSa} / \mathrm{s}$. Lines are obtained using standard sine curve fitting.

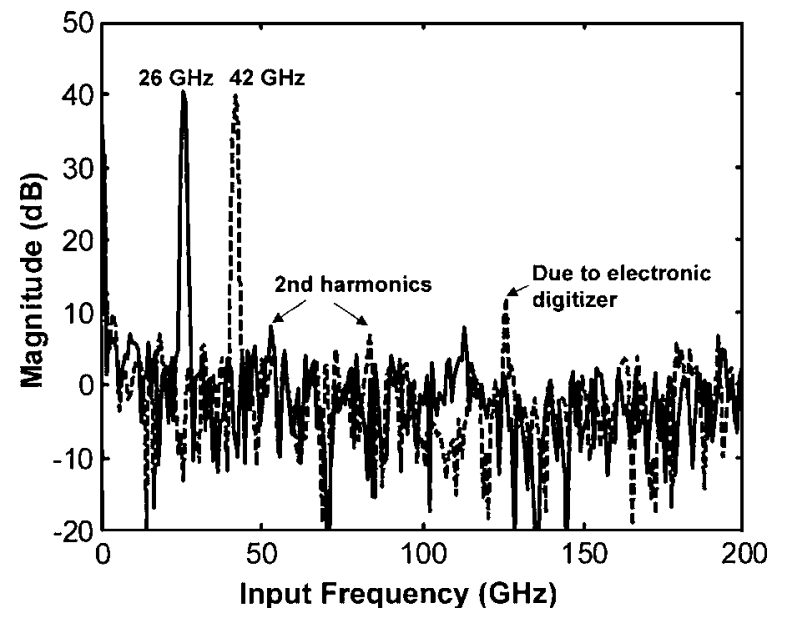

FIG. 4. The digital magnitude spectrum of captured signals at $26 \mathrm{GHz}$ (solid line) and $48 \mathrm{GHz}$ (dashed line).

the bandwidth of the electro-optic modulator to approximately $50 \mathrm{GHz}$. One method to effectively increase the modulation bandwidth is to use harmonic modulation. Conventionally, the $\mathrm{MZ}$ modulator is biased at the quadrature point, at which even harmonics are suppressed. If a MZ modulator is biased at the peak or null of transmission, fundamental frequency and odd harmonics, instead, are suppressed. The lowest frequency component is the second harmonic. Hence, an $80 \mathrm{GHz}$ sinusoid modulated optical pulse can be generated from a $40 \mathrm{GHz}$ input. The digital magnitude spectrum of captured $80 \mathrm{GHz}$ signal is shown in Fig. 5. The clean spectral line at $80 \mathrm{GHz}$ demonstrates the potential of time stretch processor beyond $50 \mathrm{GHz}$ if a $\mathrm{MZ}$ modulator with a larger bandwidth is used in the setup. The spurious tones at 40 and $120 \mathrm{GHz}$ are from the undersuppressed fundamental frequency and the third harmonic.

Optical nonlinearity may distort the stretched signal. It is especially important in $\mathrm{TSa} / \mathrm{s}$ experiment, in which multiple optical amplifications are needed to compensate fiber loss. Improper amplifier design may significantly distort signal. An example of distortion pattern is shown in Fig. 6. The distortion (amplitude reduction) is most severe at the center of pulse, where the pulse has highest peak power. This measured distortion pattern is verified by the numerical simulation and is the result of interaction between fiber dispersion and optical nonlinearity. When the optical pulse is modulated

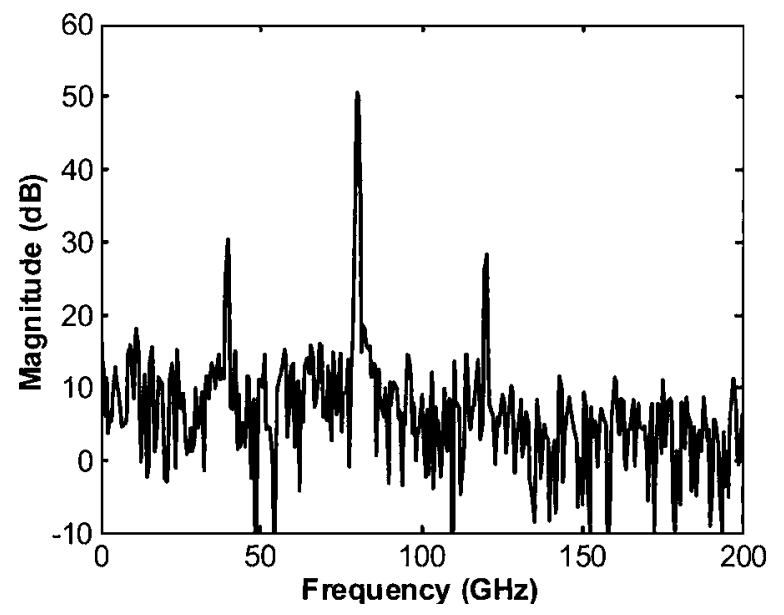

FIG. 5. The digital magnitude spectrum of captured signals at $80 \mathrm{GHz}$. 


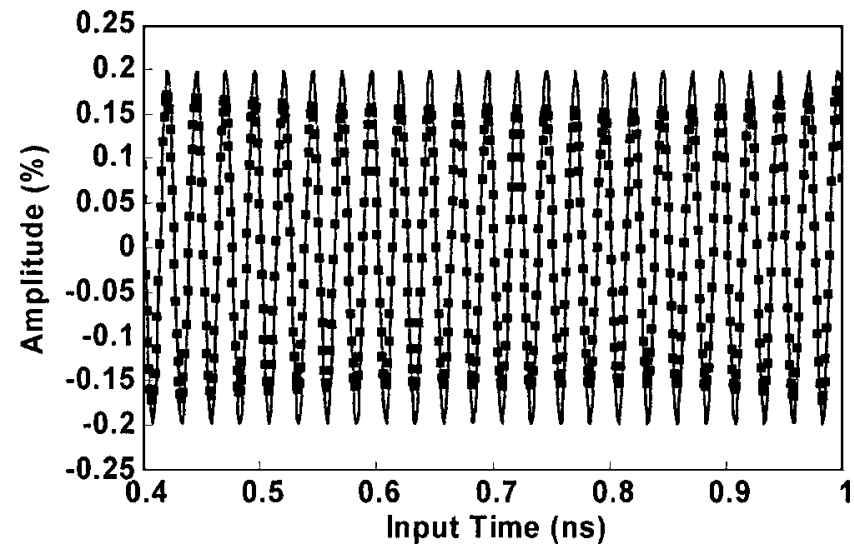

FIG. 6. Distortion caused by a nonlinear optical effect in the dispersive fiber.

by a sinusoid signal, optical nonlinearity introduces an intensity dependent phase shift in propagation. Dispersion causes phase-to-amplitude conversion and hence results in distortion of signal amplitude. ${ }^{6}$ Because optical power is highest at the center of time window, the extent of nonlinear distortion varies across the time window and introduces the distortion pattern observed in Fig. 6.

In summary, the photonic time stretch technique has been used to realize a real-time transient digitizer with the record one $\mathrm{TSa} / \mathrm{s}$ sampling rate. The system has a FWHM time aperture of $1.1 \mathrm{~ns}$. Measured over a $10 \mathrm{GHz}$ bandwidth, the ENOB of the system ranges from 3.5 to 4.2 bits depending on the input signal frequency.

This work was supported by DARPA.

${ }^{1}$ I. Wilke, A. M. MacLeod, W. A. Gillespie, G. Berden, G. M. H. Knippels, and A. F. G. van der Meer, Phys. Rev. Lett. 88, 124801 (2002).

${ }^{2}$ M. Abrams, IEEE Spectrum 40, 24 (2003).

${ }^{3}$ R. H. Walden, IEEE J. Sel. Areas Commun. 17, 539 (1999).

${ }^{4}$ W. C. Black and D. A. Hodges, IEEE J. Solid-State Circuits SC-15, 1022 (1980).

${ }^{5}$ F. Coppinger, A. S. Bhushan, and B. Jalali, Electron. Lett. 34, 399 (1998).

${ }^{6}$ Y. Han and B. Jalali, J. Lightwave Technol. 21, 3085 (2003).

${ }^{7}$ Y. Han, O. Boyraz, and B. Jalali, IEEE Trans. Microwave Theory Tech. 53, 1404 (2005) 